Pacific Journal of Mathematics

SELF-INTERSECTION NUMBER OF IMMERSIONS AND
ENUMERATION OF NONSTABLE VECTOR BUNDLES 


\title{
SELF-INTERSECTION NUMBER OF IMMERSIONS AND ENUMERATION OF NON-STABLE VECTOR BUNDLES
}

\author{
SU-WIN YANG
}

\begin{abstract}
Suppose $N$ is a closed $n$-dimensional smooth manifold and $M$ is a $2 n$-dimensional smooth manifold. In 1944, H. Whitney defined the self-intersection number for an immersion with normal crossings $f$ : $N \rightarrow M$. We show that, if $N$ is path-connected and the James-Thomas number of the normal bundle $\nu(f)$ of $f$ is greater than 1 , then the self-intersection number is completely determined by the homotopy class of $f$ and the normal bundle $\nu(f)$.
\end{abstract}

Introduction. Suppose $N$ is a closed $n$-dimensional smooth manifold and $M$ is a $2 n$-dimensional smooth manifold. For any immersion with normal crossings (having singularities of double points only and meeting itself transversally) $f: N \rightarrow M$, the set of double points $D(f)=\{f(x) \mid$ there exist $y \neq x, f(y)=f(x)\}$ is finite. In 1944, Whitney [13] defined the self-intersection number $I(f)$ :

(A) When $n$ is even and $N, M$ are oriented, $I(f)$ is an integer (algebraic sum of $D(f)$ );

(B) Otherwise, $I(f)$ is the modulo 2 reduction of number of elements in $D(f)$.

It is well-known that [Lashof and Smale, 10], in case (A), $2 I(f)$ is equal to the intersection number of the homology class $f_{*}([N])$ with itself minus the Euler number of the normal $n$-plane bundle $\nu(f)$ of $f$, where $[N] \in H_{n}(N ; Z)$ is the fundamental class of $N$. Thus, $I(f)$ is completely determined by the homology class $f_{*}([N])$ and the normal bundle $\nu(f)$. But, in case (B), $I(f)$ is not always an invariant of the homology class $f_{*}([N])$ and the normal bundle $\nu(f)$, for example, $N=S^{1}, M=\mathbf{R}^{2}$, consider the two immersions of $S^{1}$ onto the unit circle and the Figure 8, respectively.

For any $n$-plane bundle $\eta$ over $N$, the James-Thomas number of $\eta$ is the number of equivalence classes of $n$-plane bundles which are stably equivalent to $\eta$.

By Whitney [13], there is an immersion with normal crossings $f^{\prime}$ : $N \rightarrow M$ such that $f^{\prime} \simeq f$ and $I\left(f^{\prime}\right) \neq I(f)$. Now, if the James-Thomas number of $\nu(f)$ is equal to 1 , then $\nu\left(f^{\prime}\right)$ is equivalent to $\nu(f)$, and $I(f)$ 
cannot be determined by the homotopy class of $f$ and the normal bundle $\nu(f)$. The main purpose of this paper is to prove the following:

Theorem. Suppose $N$ is path-connected and the James-Thomas number of $\nu(f)$ is greater than 1. Then $I(f)$ is completely determined by the homotopy class of $f$ and the normal bundle $\nu(f)$.

Note. The James-Thomas number of $\nu(f)$ depends only on the homotopy class of $f$.

To prove the Theorem, we utilize the construction of Brown [3] and give a formula in some stable homotopy class, which is similar to the one for case (A).

We arrange the paper as follows:

1. In $\S 1$, we study the James-Thomas number (the approaches are motivated by [7]), and show that, if $N$ is path-connected and the JamesThomas number of $\eta$ is greater than 1 , then the $\mathrm{Wu}$-orientation (defined as in [2]) of $\eta$ is completely determined by the homotopy class of the map in the base spaces (detail see (1.4) and (1.7)). This was studied by Dupont [4] and earlier by Brown [3] in another version. Thus, essentially there is nothing new in this section.

2. In $\S 2$, with the help of construction given by Brown [3], we construct two stable homotopy classes in $\left\{S^{2 n+k}, T(\zeta) \wedge K\left(Z_{2}, n\right)\right\}$ ( $\zeta$ is some $k$-plane bundle) for an immersion from $N^{n}$ to $M^{2 n}$ and the difference of these two stable homotopy classes gives the self-intersection number $I(f)$. By (1.7), we have the invariance property of the two stable homotopy classes and hence that of $I(f)$ (detail see (2.6)). The proof of the formula in (2.6) depends on the technical result of (2.5), and the proof of (2.5) quite depends on the Hopf ladder, defined by Boardman and Steer [1], and the short exact sequence of homotopy classes developed by Milgram [11].

1. The James-Thomas number. Assume $\eta$ is an $n$-plane bundle over the $n$-manifold $N$. To find all $n$-plane bundles which are stably equivalent to $\eta$, we consider the spherical bundle of the Whitney sum of $\eta$ and the trivial line bundle $\varepsilon^{1}$ over $N$ and a cross-section $f: N \rightarrow S\left(\eta \oplus \varepsilon^{1}\right)$. Let $\eta(f)=\left\{v \in\left(\eta \oplus \varepsilon^{1}\right)_{x} \mid v \perp f(x), x \in N\right\}$ be the orthogonal complement of the subbundle $\{t f(x) \mid x \in N, t \in R\}$. Then, any $n$-plane bundle which is stable equivalent to $\eta$ is equivalent to $\eta(f)$ for some cross-section $f$. Furthermore, choose an embedding $\phi: D^{n} \rightarrow N$ and a local trivialization $\bar{\phi}: D^{n} \times \mathbf{R}^{n} \rightarrow V(\eta)$ such that $\pi(\bar{\phi}(x, v))=\phi(x)$, for $x \in D^{n}, v \in \mathbf{R}^{n}$, where $V(\eta)$ is the total space of $\eta$ and $\pi: V(\eta) \rightarrow N$ is the bundle 
projection. Let $\bar{\phi}_{1}: D^{n} \times \mathbf{R}^{n} \times \mathbf{R}^{1} \rightarrow V\left(\eta \oplus \varepsilon^{1}\right)$ be $\bar{\phi}_{1}(x, v, t)=(\bar{\phi}(x, v)$, $t$ ). We may assume that $\bar{\phi}_{1}$ maps $D^{n} \times S^{n}$ into $S\left(\eta \oplus \varepsilon^{1}\right)$. For each integer $i$, choose a map $d_{i}:\left(D^{n}, \partial D^{n}\right) \rightarrow\left(S^{n}, x_{0}\right)$ with degree $i$, where $x_{0}=$ $(0,0, \ldots, 0,1)$. Let $f_{l}: N \rightarrow S\left(\eta \oplus \varepsilon^{1}\right)$ be the cross-section defined by

$$
f_{l}(y)= \begin{cases}\left(s_{0}(y), 1\right), & \text { for } y \notin \phi\left(D^{n}\right), \\ \bar{\phi}_{1}\left(x, d_{i}(x)\right), & \text { for } y=\phi(x) \in \phi\left(D^{n}\right),\end{cases}
$$

where $s_{0}: N \rightarrow V(\eta)$ is the zero-section. If $N$ is path-connected, then any cross-section of $S\left(\eta \oplus \varepsilon^{1}\right)$ is homotopic to $f_{l}$ for some integer $i$, and hence $\left\{\eta\left(f_{l}\right) \mid i\right.$ : integer $\}$ consists of all the $n$-plane bundle which are stably equivalent to $\eta$. The following facts about $\left\{\eta\left(f_{l}\right)\right\}$ are standard.

Proposition (1.1). Suppose $N$ is path-connected.

(i) When $n$ is even and $V(\eta)$ is orientable, $\eta\left(f_{l}\right)$ is not equivalent to $\eta\left(f_{j}\right)$, for $i \neq j$.

(ii) If $V(\eta)$ is non-orientable, $\eta\left(f_{l+2 k}\right)$ is equivalent to $\eta\left(f_{l}\right)$, for all integers $i$ and $k$.

(iii) If $n$ is odd, $\eta\left(f_{i+2 k}\right)$ is equivalent to $\eta\left(f_{l}\right)$, for all integers $i$ and $k$.

(Proof of (1.1) is in $§ 3$.

Now we introduce a terminology which is related to the James-Thomas number.

Suppose $v$ is a cohomology class in $H^{n+1}(B O ; G)$ for some abelian group $G$.

Definition (1.2). Suppose $\xi$ is a $k$-plane bundle over $X$. For a vector bundle automorphism $\Phi$ of $\xi, \Delta_{1}(\xi, \Phi)$ is the $k$-plane bundle $\xi \times I /(v, 0)$ $\sim(\Phi(v), 1)$ over $X \times S^{1}$ (identify $I / \partial I$ as $\left.S^{1}\right)$. If $\phi: X \rightarrow O_{l}$ is a map into the orthogonal group of $\mathbf{R}^{l}$, let $\Phi: \varepsilon^{l} \rightarrow \varepsilon^{l}$ be the automorphism of the trivial $l$-plane bundle over $X$ induced from $\phi$, then we define $\Delta(\xi, \phi)$ as the $(k+l)$-plane bundle $\Delta_{1}\left(\xi \oplus \varepsilon^{l}, \operatorname{id}(\xi) \oplus \Phi\right)$, where $\operatorname{id}(\xi)$ is the identity map of $\xi$.

DeFinition (1.3). A vector bundle $\xi$ is $v$-singular, if there is a map $\phi$ : $X \rightarrow O_{l}$, such that $v(\Delta(\xi, \phi)) \neq 0$. A vector bundle $\xi$ is said to be $v$-nonsingular, if $\xi$ is not $v$-singular.

When $\operatorname{dim} \xi \geq \operatorname{dim} X+1, \xi$ is $v$-nonsingular, if and only if $v\left(\Delta_{1}(\xi, \Phi)\right)=0$, for all automorphisms $\Phi$ of $\xi$. 
Let $W_{n+1} \in H^{n+1}\left(B O ; Z_{2}\right)$ be the $(n+1)$ th Stiefel-Whitney class, we have

Proposition (1.4). Let $\eta$ be an n-plane bundle over $N$. If $\eta$ restricted to each path-component of $N$ has James-Thomas number greater than 1 , then $\eta$ is $W_{n+1}$-nonsingular.

Note. $\eta$ is $v$-nonsingular, if and only if, $\eta$ restricted to each path-component is $v$-nonsingular. It is enough to prove (1.4) for the case that $N$ is path-connected.

Proof. Assume $\eta$ is $W_{n+1}$-singular, that is, there is an automorphism $\Phi$ of $\eta \oplus \varepsilon^{1}$ such that $W_{n+1}\left(\Delta_{1}\left(\eta \oplus \varepsilon^{1}, \Phi\right)\right) \neq 0 . f_{i}: N \rightarrow S\left(\eta \oplus \varepsilon^{1}\right), i$ integers, are sections defined as above. Let $f=\Phi \circ f_{0}$. Then $\eta(f)$ is equivalent to $\eta\left(f_{0}\right)$ by $\left.\Phi\right|_{\eta\left(f_{0}\right)}$. If $f$ is homotopic to $f_{2 k}$, for some integer $k$, by non-zerosections, then we may construct a cross-section on $\Delta_{1}\left(\eta \oplus \varepsilon^{1}, \Phi\right)$ which meets the zero-section with $2 k$ points and transversally, and this implies $W_{n+1}\left(\Delta_{1}\left(\eta \oplus \varepsilon^{1}, \Phi\right)\right)=0$. Thus $f$ is homotopic to $f_{2 k+1}$ for some integer $k$, and hence, $\eta\left(f_{0}\right)$ is equivalent to $\eta\left(f_{2 k+1}\right)$. By (1.1)(i), $n$ is odd or $V(\eta)$ is non-orientable; also, by (1.1)(ii) and (iii), $\eta\left(f_{i}\right)$ is equivalent to $\eta\left(f_{j}\right)$ for all integers $i, j$. This proves (1.4).

Let $v_{n+1}$ be the $(n+1)$ th Wu class and $e_{i}$ be the class in $H^{i}\left(O ; Z_{2}\right)$ which transgresses to $W_{i+1}$ for positive integers $i$. Then there are two equivalent statements of $W_{n+1}$-nonsingular.

Proposition (1.5). (i) Suppose $N$ is a closed n-dimensional smooth manifold, $\eta$ and $\eta_{1}$ are two vector bundles over $N$ such that the Whitney sum $\eta \oplus \eta_{1}$ is equivalent to the normal bundle of $N$. Then, $\eta$ is $W_{n+1}$-nonsingular, if and only if, $\eta_{1}$ is $v_{n+1}$-nonsingular.

(ii) $A$ vector bundle $\xi$ over $X$ is $W_{n+1}$-nonsingular, if and only if, for any map $\phi: X \rightarrow O_{l}, l>\operatorname{dim} X, \Sigma_{l \geq 1} \phi^{*}\left(e_{n-l}\right) \cup W_{i}(\xi)+\phi^{*}\left(e_{n}\right)=O$. (If $i \geq$ $\left.l, e_{i}=0.\right)$

(iii) $A$ vector bundle $\xi$ over $X$ is $v_{n+1}$-nonsingular, if and only if, for any map $\phi: X \rightarrow O_{l}, l>\operatorname{dim} X, \Sigma_{s \geq 0} \phi^{*}\left(e_{2^{s}-1}\right) \cup v_{n+1-2}(\xi)=0$.

Proof. (i) Suppose $\phi: N \rightarrow O_{l}$ is a map. Consider the map $\phi^{-1}$ : $N \rightarrow O_{l}, \phi^{-1}(x)=(\phi(x))^{-1}$, then $\Delta(\eta, \phi) \oplus \Delta\left(\eta_{1}, \phi^{-1}\right)$ is equivalent to the normal bundle of $X \times S^{1}$. Thus, the Thom spaces $T(\Delta(\eta, \phi))$ and $T\left(\Delta\left(\eta_{1}, \phi^{-1}\right)\right)$ are dual each other, in Spanier-Whitehead $S$-duality. Consider their Thom classes $U_{1}$ and $U_{2}$, then $\operatorname{Sq}^{n+1}\left(U_{1}\right)=0$, if and only if, 
$\chi\left(\mathrm{Sq}^{n+1}\right)\left(U_{2}\right)=0$, that is, $W_{n+1}(\Delta(\eta, \phi))=0$, if and only if, $v_{n+1}\left(\Delta\left(\eta_{1}, \phi^{-1}\right)\right)=0$. This completes the proof.

(ii) Let $\pi_{1}: X \times S^{1} \rightarrow X$ be the projection map and $\theta(\phi)$ be the vector bundle $X \times \mathbf{R}^{l} \times I /(x, v, 0) \sim(x, \phi(x)(v), 1)$ over $X \times S^{1}$, for a map $\phi$ : $X \rightarrow O_{l}$. Then

$$
\Delta(\xi, \phi)=\pi_{1}^{\#}(\xi) \oplus \theta(\phi) .
$$

By the Whitney product formula and $W_{i+1}(\theta(\phi))=\phi^{*}\left(e_{i}\right)$, we have the result.

For (iii), it is enough to note: When $i \neq 2^{r}$, for $r$ : integer, $v_{i}(\theta(\phi))=0$; and when $i=2^{r}, v_{i}(\theta(\phi))=\phi^{*}\left(e_{i-1}\right)$.

Now, for a class $v \in H^{n+1}(B O ; G)$, we shall define the $v$-orientation as in Brown [3] and Browder [2].

Let $K$ denote the Eilenberg-Maclane space of type $(G, n+1)$, $P(K, *)$ be the path space $\{\alpha: I \rightarrow K \mid \alpha(0)=*$, the base point $\}$ and $B O\langle v\rangle=\{(x, \alpha) \in B O \times P(K, *) \mid \alpha(1)=v(x)\}$ (consider $v$ as a map from $B O$ to $K)$. Also, denote by $\zeta^{0}$ the stable vector bundle over $B O\langle v\rangle$ induced from the universal vector bundle over $B O$, by the projection $p$ : $B O\langle v\rangle \rightarrow B O, p(x, \alpha)=x$.

Definition (1.6). A $v$-orientation on a vector bundle $\eta$ over $X$ is a vector bundle map

$$
\begin{array}{rcc}
\eta & \stackrel{\bar{W}}{\rightarrow} & \zeta^{v} \\
(\bar{W}, W): \downarrow & & \downarrow \\
X & \stackrel{W}{\rightarrow} & B O\langle v\rangle
\end{array}
$$

Two $v$-orientations $\left(\bar{W}_{i}, W_{i}\right), i=0,1$, on $\eta$ are equivalent, if there is a $v$-orientation $(\bar{H}, H)$ on $\eta \times I$ such that $\bar{H}(x, i)=\bar{W}_{i}(x)$ for all $x \in \eta$ and $i=0,1$.

$\eta$ has a $v$-orientation, if and only if, $v(\eta)=0$. If two $v$-orientations $\left(\bar{W}_{l}, W_{i}\right), i=0,1$, are equivalent, then $W_{0}$ is homotopic to $W_{1}$; the converse, in general, is not true. But, we have

Proposition (1.7). Suppose a vector bundle $\eta$ over $X$ is v-nonsingular. Two v-orientations $\left(\bar{W}_{i}, W_{i}\right):(\eta, X) \rightarrow\left(\zeta^{v}, B O\langle v\rangle\right), i=0,1$ on $\eta$ are equivalent, if $W_{0}$ is homotopic to $W_{1}$.

(Proof is in §3.) 
2. Main result and its applications. First, we state the construction given by Brown [3].

In the following $\mathrm{Wu}$-orientation is the $v_{n+1}$-orientation and $\zeta$ is the vector bundle $\zeta^{v_{n+1}}$ of dimension $k$ defined in $\S 1$.

For an embedding $e: X \rightarrow \mathbf{R}^{m+k}$ of a smooth compact $m$-manifold $X$ in $\mathbf{R}^{m+k}$ with normal bundle $\nu(X)$ and a Wu-orientation on $\nu(X),(\bar{V}, V)$ : $(\nu(X), X) \rightarrow\left(\zeta, B O\left\langle v_{n+1}\right\rangle\right)$, we have the $(m+k)-S$-duality map, associated with the embedding $e, \alpha_{e}: S^{m+k} \rightarrow T(\nu(X)) \wedge(X / \partial X)$ and the map of Thom spaces induced from $\bar{V}, T(\bar{V}): T(\nu(X)) \rightarrow T(\zeta)$. Consider the map

$$
(T(\bar{V}) \wedge u) \circ \alpha_{e}: S^{m+k} \stackrel{\alpha_{e}}{\rightarrow} T(\nu(X)) \wedge(X / \partial X) \stackrel{T(\bar{V}) \wedge u}{\rightarrow} T(\zeta) \wedge Y
$$

and the associated stable homotopy class $\left\{(T(\bar{V}) \wedge u) \circ \alpha_{e}\right\}$ in $\left\{S^{m+k}, T(\zeta) \wedge Y\right\}$, where $u$ is a map from $X / \partial X$ to a space $Y$.

Proposition (2.1). Suppose $X$ and $u$ are fixed, $k>m+2$, and $\nu(X)$ is $v_{n+1}$-nonsingular. Then the stable homotopy class $\left\{(T(\bar{V}) \wedge u) \circ \alpha_{e}\right\}$ depends only on the homotopy class of $V$ in $\left[X, B O\left\langle v_{n+1}\right\rangle\right]$.

Proof. First note that, when $k>m+1, \nu(X)$ is independent of the embedding $e$.

Consider an embedding $g: X \rightarrow \mathbf{R}^{m+k}$ and a Wu-orientation of the normal bundle $\nu(g),\left(\bar{W}_{0}, W\right):(\nu(g), X) \rightarrow\left(\zeta, B O\left\langle v_{n+1}\right\rangle\right)$ such that $W$ is homotopic to $V$. We should show that $\left(T\left(\bar{W}_{0}\right) \wedge u\right) \circ \alpha_{g}$ is homotopic to $(T(\bar{V}) \wedge u) \circ \alpha_{e}$. By Haefliger [5], there is a smooth isotopy $H: X \times I \rightarrow$ $\mathbf{R}^{m+k}$ such that $H_{0}=g$ and $H_{1}=e$, where $H_{t}(x)=H(x, t)$. Extend the Wu-orientation $\left(\bar{W}_{0}, W\right)$ on $\nu(g)$ to the Wu-orientations $\left(\bar{W}_{t}, W\right)$ : $\left(\nu\left(H_{t}\right), X\right) \rightarrow\left(\zeta, B O\left\langle v_{n+1}\right\rangle\right)$, for $0 \leq t \leq 1$, continuously. Then, the maps $\left(T\left(\bar{W}_{t}\right) \wedge u\right) \circ \alpha_{H_{t}}, 0 \leq t \leq 1$, give the homotopy between $\left(T\left(\bar{W}_{0}\right) \wedge\right.$ $u) \circ \alpha_{g}$ and $\left(T\left(\bar{W}_{1}\right) \wedge u\right) \circ \alpha_{e}$. But, by (1.7) and the assumptions, the two orientations $\left(\bar{W}_{1}, W\right)$ and $(\bar{V}, V)$ are equivalent. This proves $(2.1)$.

For later application, we consider the $S$-dual of the map $T(\bar{V})$. Let $Z$ be the $(m+k+r)$-dual of $T(\zeta)$ (suppose $T(\zeta)$ has been substituted by its sufficiently high skeleton) and $\beta: Z \wedge T(\zeta) \rightarrow S^{m+k+r}$ be the $S$-duality map. Then, there is a natural isomorphism of stable homotopy classes,

$$
\beta_{*}:\left\{S^{m+k}, T(\zeta) \wedge Y\right\} \rightarrow\left\{Z, S^{r} Y\right\}
$$


(consider the two stable homotopy classes as the functors of space $Y$ ), defined by: for $g: S^{m+k} \rightarrow T(\zeta) \wedge Y$,

$$
\begin{gathered}
\beta_{*}(g)=(\beta \wedge \operatorname{id}(Y)) \circ(\operatorname{id}(Z) \wedge g): Z \wedge S^{m+k+r} \\
\stackrel{\operatorname{id} \wedge g}{\rightarrow} Z \wedge T(\zeta) \wedge Y \stackrel{\beta \wedge \text { id }}{\rightarrow} S^{m+k+r} \wedge Y,
\end{gathered}
$$

where id $(A)$ is the identity map of $A$.

Denote the map $\beta_{*}\left([T(\bar{V}) \wedge \operatorname{id}(X / \partial X)] \circ \alpha_{e}\right)$ by $\beta(e, \bar{V})$, then, by the natural property of $\beta_{*}$, we have

Proposition (2.2). $\beta_{*}\left(\left\{(T(\bar{V}) \wedge u) \circ \alpha_{e}\right\}\right)=\left\{\left(s^{r} u\right) \circ \beta(e, \bar{V})\right\}$, where $s^{r} u: S^{r}(X / \partial X) \rightarrow S^{r} Y$ is the suspension of $u$.

Now, we apply the above to immersions. Suppose $N$ is a closed smooth $n$-manifold, $M$ is a compact smooth $2 n$-manifold and $\phi: M \rightarrow$ $\mathbf{R}^{2 n+k}$ is an embedding with normal bundle $\nu(M)$. Because $v_{n+1}(\nu(M))=$ 0 , we may fixed a Wu-orientation on $\nu(M),(\bar{W}, W):(\nu(M), M) \rightarrow$ $\left(\zeta, B O\left\langle v_{n+1}\right\rangle\right)$. For an immersion $f: N \rightarrow M$, we define two stable homotopy classes in $\left\{S^{2 n+k}, T(\zeta) \wedge K\left(Z_{2}, n\right)\right\}$ as follows:

First, we choose a smooth map into the unit disk of $\mathbf{R}^{t}, f_{1}: N \rightarrow D^{t}$ such that $f \times f_{1}: N \rightarrow M \times D^{t}$ is an embedding. Consider the embedding $\phi_{1}: M \times D^{t} \rightarrow \mathbf{R}^{2 n+k+t}, \phi_{1}(x, y)=(\phi(x), y) \in \mathbf{R}^{2 n+k} \times \mathbf{R}^{t}$ and the Wu-orientation $\left(\bar{W}_{1}, W_{1}\right):\left(\nu\left(M \times D^{t}\right), M \times D^{t}\right) \rightarrow\left(\zeta, B O\left\langle v_{n+1}\right\rangle\right)$ on $\nu\left(M \times D^{t}\right)=\nu(M) \times D^{t}$ induced from $(\bar{W}, W)$.

(i) Let $u_{0}: M / \partial M \rightarrow K\left(Z_{2}, n\right)$ represent the cohomology class which is the Poincaré dual of $f_{*}([N])$ and

$$
u_{1}=u_{0} \wedge \mathrm{id}\left(D^{t} / \partial D^{t}\right): M \times D^{t} / \partial\left(M \times D^{t}\right) \rightarrow K\left(Z_{2}, n\right) \wedge S^{t} .
$$

Define $\Phi(f, N, M, \bar{W})=\left\{\left(T\left(\bar{W}_{1}\right) \wedge u_{1}\right) \circ \alpha_{\phi_{1}}\right\}$

(ii) Let $\eta$ denote the normal bundle of $f$ and $E(\eta)$ the disk bundle of $\eta$. Extend $f$ to an immersion on $E(\eta), \bar{f}: E(\eta) \rightarrow M$; define $\tilde{f}: E(\eta) \times D^{t}$ $\rightarrow M \times \mathbf{R}^{t}$ by $\tilde{f}(x, y)=\left(\bar{f}(x), f_{1}(\pi(x))+y\right)$ and $\tilde{f}_{\lambda}: E(\eta) \times D^{t} \rightarrow M \times$ $\mathbf{R}^{t}$ by $\tilde{f}_{\lambda}(x, y)=(\lambda x, \lambda y)$, for positive number $\lambda \leq 1$, where $\pi: E(\eta) \rightarrow N$ is the bundle projection. By the choice of $f_{1}, \tilde{f}_{\lambda}$ is an embedding for small $\lambda$. We substitute $\tilde{f}_{\lambda}$ for $\tilde{f}$ for a small positive $\lambda$. Then $E(\eta) \times D^{t}$ is embedded in $\mathbf{R}^{2 n+k+t}$ (by $\left.\phi_{1} \circ \tilde{f}\right)$ with normal bundle $\tilde{f}^{\sharp}\left(\nu\left(M \times D^{t}\right)\right)$, and we denote by $\tilde{f}^{\sharp}\left(\bar{W}_{1}\right)$ the induced Wu-orientation on $\tilde{f}^{\sharp}\left(\nu\left(M \times D^{t}\right)\right)$. Let $U: E(\eta) / \partial(E(\eta)) \rightarrow K\left(Z_{2}, n\right)$ be the Thom class and $U_{1}=U \wedge \operatorname{id}\left(S^{t}\right)$.

Define

$$
\Psi(f, N, M, \bar{W})=\left\{\left[\left(T \tilde{f}^{\sharp}\left(\bar{W}_{1}\right)\right) \wedge U_{1}\right] \circ \alpha_{\left(\phi_{1} \circ \tilde{f}\right)}\right\} .
$$


The stable homotopy classes are actually independent of the choice of $f_{1}$. Under some assumption, we have stronger results.

Proposition (2.3). (i) If the two immersions $f, g: N \rightarrow M$ are homotopic, then $\Phi(f, N, M, \bar{W})=\Phi(g, N, M, \bar{W})$.

(ii) Suppose $f^{\#}(\nu(M))$ is $v_{n+1}$-nonsingular. If the two immersions $f, g$ : $N \rightarrow M$ are homotopic and the normal bundles $\nu(f)$ and $\nu(g)$ are equivalent, then $\Psi(f, N, M, \bar{W})=\Psi(g, N, M, \bar{W})$.

(iii) Suppose $g_{1}: N \rightarrow M_{1}, g_{2}: N \rightarrow M_{2}$ are two immersions, $\operatorname{dim} M_{l}=$ $2 n, i=1,2$, the James-Thomas number of $\nu\left(g_{1}\right)$ is greater than 1 and $\left(\bar{W}_{i}, W_{i}\right):\left(\nu\left(M_{i}\right), M_{i}\right) \rightarrow\left(\zeta, B O\left\langle v_{n+1}\right\rangle\right), i=1,2$, are Wu-orientations. If $\nu\left(g_{1}\right)$ is equivalent to $\nu\left(g_{2}\right)$ and $W_{1} \circ g_{1}$ is homotopic $W_{2} \circ g_{2}$, then $\Psi\left(g_{1}, N, M_{1}, \bar{W}_{1}\right)=\Psi\left(g_{2}, N, M_{2}, \bar{W}_{2}\right)$.

Proof. (i) follows the definition directly. By (2.1), we have (ii). For (iii): By (1.4), $\nu\left(g_{1}\right)$ is $W_{n+1}$-nonsingular. $\nu\left(g_{1}\right) \oplus g_{1}^{\#}\left(\nu\left(M_{1}\right)\right)$ is equivalent to $\nu(N)$, by $(1.5), g_{1}^{\sharp}\left(\nu\left(M_{1}\right)\right)$ is $v_{n+1}$-nonsingular. Hence, by (2.1), we have (iii).

Proposition (2.4). (i) $\beta_{*}(\Phi(f, N, M, \bar{W}))=\left\{s^{r} u_{1} \circ \beta\left(\phi_{1}, \bar{W}_{1}\right)\right\}$.

(ii)

$$
\beta_{*}(\Psi(f, N, M, \bar{W}))=\left\{s^{r} U_{1} \circ s^{r} \tau(\tilde{f}) \circ \beta\left(\phi_{1}, \bar{W}_{1}\right)\right\}
$$

where $\tau(\tilde{f}): M / \partial M \wedge S^{t} \rightarrow(E(\eta) / \partial E(\eta)) \wedge S^{t}$ is the Thom-Pontrjagin construction associated with the embedding $\tilde{f}$.

Proof. By (2.2), we have (i) and

$$
\beta_{*}(\Psi(f, N, M, \bar{W}))=\left\{s^{r} U_{1} \circ \beta\left(\phi_{1} \circ \tilde{f}, \tilde{f}^{\sharp}\left(\bar{W}_{1}\right)\right)\right\} .
$$

Thus, we need to show $\beta\left(\phi_{1} \circ \tilde{f}, \tilde{f}^{\sharp}\left(\bar{W}_{1}\right)\right)=s^{r} \tau(\tilde{f}) \circ \beta\left(\phi_{1}, \bar{W}_{1}\right)$. Let $\tilde{f}^{\sharp}$ denote the inclusion $\tilde{f}^{\sharp}\left(\nu\left(M \times D^{t}\right)\right) \rightarrow \nu\left(M \times D^{t}\right)$, then $T\left(\tilde{f}^{\sharp}\left(\bar{W}_{1}\right)\right)=$ $T\left(\bar{W}_{1}\right) \circ T\left(\tilde{f}^{\sharp}\right)$, and, by the definition of Thom construction, we have the equality

$$
(T(\tilde{f} \sharp) \wedge \mathrm{id}) \circ \alpha_{\left(\phi_{1} \circ \tilde{f}\right)}=(\mathrm{id} \wedge \tau(\tilde{f})) \circ \alpha_{\phi_{1}} .
$$$$
\beta\left(\phi_{1} \circ \tilde{f}, \tilde{f}^{\sharp}\left(\bar{W}_{1}\right)\right)=\beta_{*}\left(\left[T\left(\tilde{f}^{\sharp}\left(\bar{W}_{1}\right)\right) \wedge \mathrm{id}\right] \circ \alpha_{\left(\phi_{1} \circ \tilde{f}\right)}\right) \quad \text { (by definition) }
$$

$$
\begin{aligned}
& =\beta_{*}\left(\left[T\left(\bar{W}_{1}\right) \wedge \mathrm{id}\right] \circ\left[T\left(\tilde{f}^{\#}\right) \wedge \mathrm{id}\right] \circ \alpha_{\left(\phi_{1} \circ \tilde{f}\right)}\right) \\
& =\beta_{*}\left(\left[T\left(\bar{W}_{1}\right) \wedge \mathrm{id}\right] \circ(\mathrm{id} \wedge \tau(\tilde{f})) \circ \alpha_{\phi_{1}}\right) \quad(\text { by }(*)) \\
& =\beta_{*}\left(\left[T\left(\bar{W}_{1}\right) \wedge \tau(\tilde{f})\right] \circ \alpha_{\phi_{1}}\right)=s^{r} \tau(\tilde{f}) \circ \beta\left(\phi_{1}, \bar{W}_{1}\right) \quad(\text { by }(2.2)) .
\end{aligned}
$$


Proposition (2.5). If $f: N \rightarrow M$ is an immersion with normal crossing, $f_{1}$ can be chosen as a map from $N$ into $\mathbf{R}^{\mathbf{l}}(t=1)$. Then

$$
u_{1}-U_{1} \circ \tau(\tilde{f})=I(f) \mu_{1} \text { in }\left[S M / \partial M, S K\left(Z_{2}, n\right)\right] \text {, }
$$

where $\mu_{1} \in\left[S M / \partial M, S K\left(Z_{2}, n\right)\right]$ is the composite of the generator $\mu_{0}$ of $\left[S^{2 n+1}, S K\left(Z_{2}, n\right)\right]$ and the map of degree 1 from $S M / \partial M$ to $S^{2 n+1}$.

(Proof of (2.5) is in $§ 3$. )

Combining (2.3), (2.4) and (2.5), we have the following main result.

THEOREM (2.6). Suppose $N$ is a closed $n$-dimensional smooth manifold, $M$ is a compact $2 n$-dimensional smooth manifold, $\phi: M \rightarrow \mathbf{R}^{2 n+k}$ is an embedding with normal bundle $\nu(M)$, and $(\bar{W}, W):(\nu(M), M) \rightarrow$ $\left(\zeta, B O\left\langle v_{n+1}\right\rangle\right)$ is a Wu-orientation on $\nu(M)$. Then, for any immersion with normal crossings $f: N \rightarrow M$, the stable homotopy classes $\Phi(f, N, M, \bar{W})$ and $\Psi(f, N, M, \bar{W})$ are defined and the following equality holds

$$
\Phi(f, N, M, \bar{W})-\Psi(f, N, M, \bar{W})=I(f) \mu
$$

where $\mu=\beta_{*}^{-1}\left(s^{t+r-1} \mu_{1} \circ \beta\left(\phi_{1}, \bar{W}_{1}\right)\right)$ is an element of order 2. If $f^{\sharp}(\nu(M))$ is $v_{n+1}$-nonsingular, then the left-hand side of the above equality is completely determined by the homotopy class of $f$ and the normal bundle of $f$, and hence so does the modulo 2 reduction of $I(f)$.

REMARK. (i) In (2.6), I( $f$ ) can be substituted by the number of double points in $M$.

(ii) Let $g: S^{k} \rightarrow T(\zeta)$ be the inclusion of $S^{k}$ as a fibre in $T(\zeta)$, and

$$
g_{*}:\left\{S^{2 n+k}, S^{k} \wedge K\left(Z_{2}, n\right)\right\} \rightarrow\left\{S^{2 n+k}, T(\zeta) \wedge K\left(Z_{2}, n\right)\right\}
$$

be the induced map. Denote by $\mu_{0}$ the generator of $\left\{S^{2 n+k}, S^{k} \wedge\right.$ $\left.K\left(Z_{2}, n\right)\right\}$. Then

$$
g_{*}\left(\mu_{0}\right)=\mu
$$

Corollary (2.7). Suppose $N$ is a closed $n$-dimensional smooth manifold and $f: N \rightarrow M$ is an immersion with normal crossings into a $2 n$-dimensional smooth manifold. Then, the following statements are equivalent:

(i) The normal bundle of $f$ restricted to each path-component of $N$ has James-Thomas number greater than 1 .

(ii) The normal bundle of $f$ is $W_{n+1}$-nonsingular.

(iii) $f^{\sharp}(\nu(M))$ is $v_{n+1}$-nonsingular. 
(iv) If $g: N \rightarrow M$ is an immersion with normal crossings satisfyin the following two conditions:

(1) $g$ is homotopic to $f$,

(2) the normal bundle of $f$ and $g$ are equivalent, then $I(g)=I(f)$ $(\bmod 2)$.

Proof. We need only to show that (iii) implies (iv). Assume (iii) and the assumption in (iv). Consider a compact $2 n$-submanifold $M^{\prime}$ of $M$ such that the homotopy of $f$ and $g$ is contained in $M^{\prime}$. Then, by (2.6), $I(f)=I(g)$.

The following two applications directly follow (2.7) and (1.5).

Corollary (2.8). If the dimension of $N$ is not equal to $2^{\imath}-1$, for any integer $i$ and $M$ is almost parallelizable, then $f^{\sharp}(\nu(M))$ is $v_{n+1}$-nonsingular and hence the statement (iv) in (2.7) holds.

Corollary (2.9). Suppose, for any map $\phi: N \rightarrow 0, \phi^{*}\left(e_{n}\right)=0$. Then, for any two homotopic immersions $f, g: N \rightarrow M$ with trivial normal bundle, $I(f)=I(g)$.

\section{Proofs.}

Proof of (1.1). (i) When $n$ is even and $V(\eta)$ is orientable, the Euler number $\chi(\eta)$ of $\eta$ is defined and $\chi\left(\eta\left(f_{i+1}\right)\right)=\chi\left(\eta\left(f_{l}\right)\right)+2$. This implies the result.

(ii) If $V(\eta)$ is non-orientable, then $f_{l+2 k}$ is homotopic to $f_{l}$, for all integers $i$ and $k$.

(iii) First, we define the connected sum of two vector bundles. Suppose $M_{l}, i=1,2$ are $m$-manifold, $\eta_{l}$ are $k$-plane bundles over $M_{l}$, $i=1,2$, and $\theta_{i}: D^{m} \times \mathbf{R}^{k} \rightarrow \eta_{t}, i=1,2$ are local trivializations. The connected sum $\eta_{1} \# \eta_{2}$ is defined as the $k$-plane bundle

$$
\begin{array}{r}
{\left[\eta_{1}-\theta_{1}\left(\stackrel{\circ}{ }^{m} \times \mathbf{R}^{k}\right)\right] \cup\left[\eta_{2}-\theta_{2}\left(\stackrel{\circ}{D^{m}} \times \mathbf{R}^{k}\right)\right] / \theta_{1}(x, v) \sim \theta_{2}(x, v),} \\
x \in S^{m-1}, \quad v \in \mathbf{R}^{k},
\end{array}
$$

over $M_{1} \# M_{2}$.

By the definition of $f_{l}$, we have $\eta\left(f_{t+1}\right)=\eta\left(f_{i}\right) \# \tau\left(S^{n}\right)$, where $\tau\left(S^{n}\right)$ is the tangent bundle of $S^{n}$. But, $\tau\left(S^{n}\right) \# \tau\left(S^{n}\right)$ is a trivial vector bundle, this proves (1.1). 
Proof of (1.7). Let $\bar{p}: \zeta^{0} \rightarrow \zeta_{0}$ denote the vector bundle map covering $p$ and let $\bar{g}=\bar{p} \circ \bar{W}_{0}, g=p \circ W_{0}$. By the assumption that $W_{0}$ is homotopic to $W_{1}$, there is a Wu-orientation $\left(\bar{W}_{0}^{\prime}, W_{0}\right)$ on $\eta$ which is equivalent to $\left(\bar{W}_{1}, W_{1}\right)$. Comparing the two bundle map $\bar{W}_{0}$ and $\bar{W}_{0}^{\prime}$, there is an automorphism $\Phi$ of $\eta$ such that $\bar{W}_{0}^{\prime}=\bar{W}_{0} \circ \Phi$. Consider the vector bundle $\Delta_{1}(\eta, \Phi)$, let $(\bar{\theta}, \theta):\left(\Delta_{1}(\eta, \Phi), X \times S^{\prime}\right) \rightarrow\left(\zeta_{0}, B O\right)$ be a classifying map of $\Delta_{1}(\eta, \Phi)$ such that $(\bar{\theta} \circ \bar{j})(y, 0)=\bar{g}(y),(\theta \circ j)(x, 0)=g(x)$, for $y \in \eta$, $x \in X$, where $(\bar{j}, j):(\eta \times I, X \times I) \rightarrow\left(\Delta_{1}(\eta, \Phi), X \times S^{1}\right)$ is the identification map in the construction of $\Delta_{1}$. Let $(\bar{h}, h)=(\bar{\theta}, \theta) \circ(\bar{j}, j):(\eta \times$ $I, X \times I) \rightarrow\left(\zeta_{0}, B O\right)$, then $h_{0}=h_{1}=g, \bar{h}_{0}=\bar{g}$ and $\bar{h}_{1} \circ \Phi=\bar{g}$. Furthermore, by assumption that $\eta$ is $v$-nonsingular; $v \circ \theta: X \times S^{1} \rightarrow K$ is nullhomotopic; this implies that there is a homotopy $H: X \times I \times I \rightarrow K$ such that $H\left(x, s_{1}, 0\right)=v\left(h\left(x, s_{1}\right)\right)$ and $H\left(x, 0, s_{2}\right)=H\left(x, 1, s_{2}\right)=H\left(x, s_{1}, 1\right)$ $=v(g(x))$, for all $s_{1}, s_{2}$ in $[0,1]$ and $x$ in $X$. Let $\alpha: X \rightarrow K^{I}$ be the map such that $W_{0}(x)=(g(x), \alpha(x))$, for all $x \in X$, and consider the maps $H_{s_{1}, s_{2}}: X \rightarrow K^{I}, H_{s_{1}, s_{2}}(x)(t)=H\left(x, s_{1} t, s_{2}\right)$, then $\alpha(x)(1)=v(g(x))=$ $H_{s_{1}, s_{2}}(x)(0)$ and we have the map $\alpha * H_{s_{1}, s_{2}}: X \rightarrow K^{I},\left(\alpha * H_{s_{1}, s_{2}}\right)(x)=$ the path product of $\alpha(x)$ and $H_{s_{1}, s_{2}}(x)$. When $\left(s_{1}, s_{2}\right)$ is in the path-connected set $C=[0,1] \times\{0\} \cup\{1\} \times[0,1],\left(\alpha * H_{s_{1}, s_{2}}\right)(x)(1)=v\left(h\left(x, s_{1}\right)\right), W_{s_{1}, s_{2}}$ $=\left(h_{s_{1}}, \alpha * H_{s_{1}, s_{2}}\right): X \rightarrow B O\langle v\rangle$ is a well-defined map. For $\left(s_{1}, s_{2}\right) \in C$, let $\bar{W}_{s_{1}, s_{2}}: \eta \rightarrow \zeta^{0}$ be the unique vector bundle map such that $\bar{W}_{s_{1}, s_{2}}$ covers $W_{s_{1}, s_{2}}$ and $\bar{p} \circ \bar{W}_{s_{1}, s_{2}}=\bar{h}_{s_{1}}$. Then $\bar{W}_{0,0}$ is equivalent to $\bar{W}_{1,1}$ and $\bar{W}_{1,1}=$ $\bar{W}_{0,0} \circ \Phi^{-1}$. But, it is easy to see that $\bar{W}_{0,0}$ is equivalent to $\bar{W}_{0}$. This shows that $\bar{W}_{0}^{\prime}$ is equivalent to $\bar{W}_{0}$.

Proof of (2.5). Let $L=M / \partial M$. By Brown [3] or Milgram [11], there is a short exact sequence

$$
0 \rightarrow H^{2 n}\left(L ; Z_{2}\right) \stackrel{j}{\rightarrow}\left[S L, S K\left(Z_{2}, n\right)\right] \stackrel{\Delta}{\rightarrow} H^{n}\left(L ; Z_{2}\right) \rightarrow 0
$$

where $j\left(x_{0}\right)=\mu_{1}$, for $x_{0}$ the generator of $H^{2 n}\left(L ; Z_{2}\right) ; \Delta$ is defined by: for $g: S L \rightarrow S K\left(Z_{2}, n\right), \Delta(g)=s^{-1}\left(g^{*}(A)\right), A$ is the fundamental class of $S K(Z, n)$ in $H^{n+1}\left(S K\left(Z_{2}, n\right) ; Z_{2}\right)$ and $s$ is the suspension isomorphism in cohomology.

Thus, $\Delta\left(U_{1} \circ \tau(\tilde{f})\right)=u_{0}, \Delta\left(u_{1}\right)=u_{0}$, and hence, $U_{1} \circ \tau(\tilde{f})-u_{1}=$ $m \mu_{1}$, for some integer $m$.

Consider the Hopf ladder $\lambda_{2}$ of degree 2, defined by Boardman and Steer [1], $\lambda_{2}:[S L, S Y] \rightarrow\left[S^{2} L, S Y \wedge S Y\right]$. By the natural property of $\lambda_{2}$, 
$U: T(\eta) \rightarrow K\left(Z_{2}, n\right)$ induces a commutative diagram

$$
\begin{array}{ccc}
{[S L, S T(\eta)]} & \stackrel{\lambda_{2}}{\rightarrow} & {\left[S^{2} L, S T(\eta) \wedge S T(\eta)\right]} \\
\downarrow s U_{*} & & \downarrow(s U \wedge s U)_{*} \\
{\left[S L, S K\left(Z_{2}, n\right)\right]} & \stackrel{\lambda_{2}}{\rightarrow} & {\left[S^{2} L, S K\left(Z_{2}, n\right) \wedge S K\left(Z_{2}, n\right)\right]} \\
& & {\left[L, K\left(Z_{2}, 2 n\right)\right]}
\end{array}
$$

Let $x_{1}$ be the generator of $\left[S^{2} L, S T(\eta) \wedge S T(\eta)\right]\left(\approx Z\right.$ or $\left.Z_{2}\right)$, then $(s U \wedge s U)_{*}\left(x_{1}\right)=x_{0}$. By the result of Koschorke and Sanderson [8], $\lambda_{2}(\tau(\tilde{f}))=I(f) x_{1}$, and hence $\lambda_{2}\left(U_{1} \circ \tau(\tilde{f})\right)=I(f) x_{0}$. Also, by the definition of Hopf ladder and $u_{1}$ is the suspension of $u_{0}, \lambda_{2}\left(u_{1}\right)=0$. For any maps $g: S L \rightarrow S B$ and $h: S L \rightarrow S C$, let $g \cdot h=(g \wedge h) \circ\left(s^{2} d\right): S^{2} L \rightarrow$ $S B \wedge S C$ denote the cup product, as in [1], where $d: L \rightarrow L \wedge L$ is the reduced diagonal, then we have $\lambda_{2}(g+h)=\lambda_{2}(g)+g \cdot h+\lambda_{2}(h)$. Let $g_{1}: S L \rightarrow S^{2 n+1}$ be the map of degree 1 and $\mu_{0} \in\left[S^{2 n+1}, S K\left(Z_{2}, n\right)\right]$ be the generator, then $\mu_{1}=\mu_{0} \circ g_{1}$ and $\mu_{1} \cdot u_{1}=\left(\mu_{0} \wedge \mathrm{id}\right) \circ\left(g_{1} \cdot u_{1}\right)$. But, $g_{1} \cdot u_{1}$ maps $S^{2} L$ into $S^{2 n+1} \wedge S K\left(Z_{2}, n\right)$ which is $3 n$-connected; when $n \geq 1, g_{1} \cdot u_{1}=0$. Thus, we have $\lambda_{2}\left(m \mu_{1}+u_{1}\right)=\lambda_{2}\left(m \mu_{1}\right)+\lambda_{2}\left(u_{1}\right)=$ $m \lambda_{2}\left(\mu_{1}\right) \cdot \mu_{1}$ is of order $2, U_{1} \circ \tau(\tilde{f})=m \mu_{1}+u_{1}$, hence we have $I(f) x_{0}=$ $m \lambda_{2}\left(\mu_{1}\right)$. Because there is some immersion $f$ such that $I(f) \neq 0, \lambda_{2}\left(\mu_{1}\right)=$ $x_{0}$ and hence $I(f) \equiv m(\bmod 2)$.

\section{REFERENCES}

[1] J. M. Boardman and B. Steer, On Hopf invariants, Comment. Math. Helvet., 42 (1967), 180-221.

[2] W. Browder, The Kervaire invariant of framed manifold and its generalization, Ann. Math., 90 (1969), 157-186.

[3] E. H. Brown, Generalizations of the Kervaire invariant, Ann. Math., 95 (1972), 368-383.

[4] J. L. Dupont, On the homotopy invariance of the tangent bundle II, Math. Scand., 26 (1970), 200-220.

[5] A. Haefliger, Differentiable imbeddings, Bull. Amer. Math. Soc., 67 (1961), 109-112.

[6] M. W. Hirsch, Immersions of manifolds, Trans. Amer. Math. Soc., 93 (1959), 504-537.

[7] I. M. James and E. Thomas, An approach to the enumeration problem for non-stable vector bundles, J. Math. Mech., 14 (1965), 485-506.

[8] U. Koschorke and B. Sanderson, Self-intersections and higher Hopf invariant, Topology, 17 (1978), 283-290.

[9] U. Koschorke and B. Sanderson, Geometric interpretations of the generalized Hopf invariants, Math. Scand., 41 (1977), 199-217. 
[10] R. K. Lashof and S. Smale, Self-intersections of Immersed manifolds, J. Math. Mech., 8 (1959), 143-157.

[11] R. J. Milgram, Surgery with coefficients, Ann. Math., 100 (1974), 194-248.

[12] W. A. Sutherland, The Browder-Dupont invariant, Proc. London Math. Soc., (3) 33 (1976), 94-112.

[13] H. Whitney, The Self-intersections a smooth n-manifold in $2 n$-spaces, Ann. Math., (1944), 220-240.

Received June 30, 1982.

DePartment of Mathematics

NATIONAL TAIWAN UNIVERSITY

TAIPEI, TAIWAN 



\section{PACIFIC JOURNAL OF MATHEMATICS \\ EDITORS}

Donald BABBITT (Managing Editor)

University of California

Los Angeles, CA 90024

Hugo Rossi

University of Utah

Salt Lake City, UT 84112

C. C. Moore and Arthur Ogus

University of California

Berkeley, CA 94720
J. DugunduI

Department of Mathematics

University of Southern California

Los Angeles, CA 90089-1113

R. FinN and H. SAMELSON

Stanford University

Stanford, CA 94305

ASSOCIATE EDITORS

R. ARENS

E. F. BECKENBACH

B. H. NeUMANN

F. WOLF

K. YosHIDA (1906-1982)

\section{SUPPORTING INSTITUTIONS}

UNIVERSITY OF ARIZONA

UNIVERSITY OF BRITISH COLUMBIA

CALIFORNIA INSTITUTE OF TECHNOLOGY

UNIVERSITY OF CALIFORNIA

MONTANA STATE UNIVERSITY

UNIVERSITY OF NEVADA, RENO

NEW MEXICO STATE UNIVERSITY

OREGON STATE UNIVERSITY
UNIVERSITY OF OREGON

UNIVERSITY OF SOUTHERN CALIFORNIA

STANFORD UNIVERSITY

UNIVERSITY OF HAWAII

UNIVERSITY OF TOKYO

UNIVERSITY OF UTAH

WASHINGTON STATE UNIVERSITY

UNIVERSITY OF WASHINGTON 


\section{Pacific Journal of Mathematics}

\section{Vol. 113, No. $1 \quad$ March, 1984}

Flavio E. A. da Silveira, Rational homotopy theory of fibrations $\ldots \ldots \ldots \ldots 1$

Donald M. Davis, Desuspensions of stunted projective spaces ............ 35

Lou van den Dries, Exponential rings, exponential polynomials and

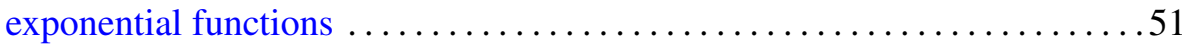

Fred Galvin and Samuel David Shore, Completeness in semimetric

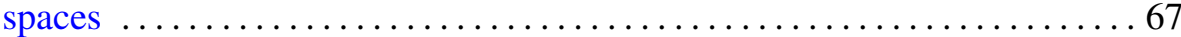

Fereidoun Ghahramani, Compact elements of weighted group algebras . . . 777

Munehiko Itōo, The closed image of a hereditary $M_{1}$-space is $M_{1} \ldots \ldots \ldots 85$

Elvira Laura Livorni, Classification of algebraic surfaces with sectional genus less than or equal to six. I. Rational surfaces .............. 93

H. Alan MacLean, Riesz sets and a theorem of Bochner ............ 115

E. Neher, Jordan triple systems with completely reducible derivation or

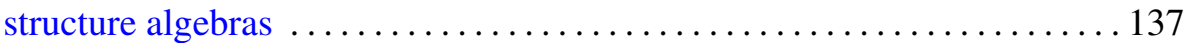

Joe Repka, Shalika's germs for $p$-adic GL( $n)$. I. The leading term $\ldots \ldots \ldots 165$

Joe Repka, Shalika's germs for $p$-adic GL( $n)$. II. The subregular term $\ldots \ldots 173$

Rae Michael Andrew Shortt, Borel density, the marginal problem and isomorphism types of analytic sets $\ldots \ldots \ldots \ldots \ldots \ldots \ldots \ldots \ldots \ldots \ldots \ldots \ldots$

Baruch Solel, The multiplicity functions of invariant subspaces for nonselfadjoint crossed products $\ldots \ldots \ldots \ldots \ldots \ldots \ldots \ldots \ldots \ldots \ldots \ldots . \ldots \ldots$

Su-win Yang, Self-intersection number of immersions and enumeration of nonstable vector bundles

W. M. Zajączkowski, Local solvability of nonstationary leakage problem for ideal incompressible fluid. II 